\title{
Air Quality Prediction of Pollution Sources Based on Wavelet Neural Network
}

\author{
Hong-Da Ji ${ }^{1, a}$, Li Yang ${ }^{2, b}$, Yan-Feng Wang ${ }^{3, c}$, Xiu-Bin Dai ${ }^{2, d}$ and Jun Wang ${ }^{2, e}$ \\ ${ }^{1}$ Image Processing and Image Communications Key Lab, Nanjing University of Posts \& Telecomm \\ Nanjing, China \\ ${ }^{2}$ Image Processing and Image Communications Key Lab, Nanjing University of Posts \& Telecomm \\ Nanjing, China \\ ${ }^{3}$ Environmental Protection Department of Jiangsu Province, Nanjing, China \\ a1345267276@qq.com, byangli@njupt.edu.cn, cwyf@jshb.gov.cn, ddaixb@njupt.edu.cn, \\ ewangj@njupt.edu.cn
}

Keywords: wavelet neural network, pollution source, air quality, forecasting.

\begin{abstract}
In order to realize the prediction of air quality of pollution sources, this paper uses the method of wavelet neural network. In this paper, the pollutant concentration values of pollution sources were predicted, so as to realize the monitoring and early warning of pollution source emission. In this paper, the main chemical plants in Taizhou city are selected as the research object. The wavelet neural network is used to forecast the concentration of sulfur dioxide, nitrogen oxide of the chemical plant emissions. Finally, the experiments show that the wavelet neural network is useful to forecast the concentration of pollution sources.
\end{abstract}

\section{Introduction}

In recent years, more and more people pay attention to the air quality problems. With the rapid development of Jiangsu Province, how to improve the air quality has become the focus of people's attention. In the past, most researches on air quality in a city were conducted, and the research on the value of pollutant concentration of pollution source was carried out [1]. Air quality monitoring and early warning from the pollution source, it is conducive to the discovery of the root causes of air pollution, to establish the city air quality prediction system to provide the basis for. The prediction of air quality from pollution sources provides a scientific basis for the management of air quality in the environmental management department. Therefore, it is very important to monitor the air quality of the pollution sources. At present, the time series, regression analysis, grey system and so on are used to predict the air quality at home and abroad. But the accuracy of these methods is not very high [2]. Because the change of the concentration of air pollutants is influenced by many factors, so that the characteristics of nonlinear. So the prediction of pollutant concentration is a nonlinear problem, which can not be used to determine the mathematical model [3].

Studies at home and abroad show that the neural network can better predict the pollutant concentration than the time series analysis, regression analysis and so on [4]. The wavelet neural network which is proposed in this paper is a research method which combines the theory of neural network and wavelet theory. Wavelet neural network has the advantages of neural network self-learning, adaptive, robustness and fault tolerance, and has the characteristics of time frequency local characteristics of wavelet transform [5]. The research shows that the wavelet neural network is applied to all fields of neural network research, which can solve the nonlinear problem well, so the wavelet neural network is widely used in the study of nonlinear problems [6].

\section{Wavelet neural network model}

\subsection{Wavelet Theory}

The wavelet analysis is made up for the defects of Fourier transform. In the field of signal 
processing, Fourier transform is an analysis of the most widely used method, but Fourier transform has some obvious shortcomings, it does not contain time information, also shows that the Fourier transform can not judge the signal of the time, Fourier transform cannot be applied to the field of time [7]. Wavelet is a mean value of the waveform, and its length is limited. Wavelet has the following characteristics:

a. Time domain has compact support or approximate compact support;

b. DC component is 0 ;

The wavelet function is obtained by translation and size scaling of the mother wavelet function. Wavelet analysis is a method of decomposing a signal into a number of wavelet functions.

$\psi(t)$ is basic wavelet function, $x(\mathrm{t})$ is the signal to be analyzed. Wavelet analysis is that $\psi(t)$ translates $\tau$, and then makes inner product with $x(\mathrm{t})$ in different scale a.

$$
f_{x}(a, \tau)=\frac{1}{\sqrt{a}} \int_{-\infty}^{\infty} x(t) \psi\left(\frac{t-\tau}{a}\right) d t \quad a>0
$$

The equivalent time domain expression is:

$$
f_{x}(a, \tau)=\frac{1}{\sqrt{a}} \int_{-\infty}^{\infty} x(\omega) \psi(a \omega) e^{j \omega} d t \quad a>0
$$

In the equation, $\tau$ and $a$ are the parameters. The function of $\tau$ is to make horizontal movement of the camera relative to the target. The function of $a$ is to push or far away from the camera. From the formula (1) and formula (2), we can see that wavelet analysis can be used to analyze the local characteristics of the signal by wavelet transform. In two dimensional case the direction of the signal can be selected.

\subsection{Wavelet Neural Network}

Wavelet neural network is based on the BP neural network topology, the wavelet basis function as the transfer function of the hidden layer of BP neural network, the forward propagation of the signal at the same time, the error back propagation. The topological structure of wavelet neural network is shown in figure 1:

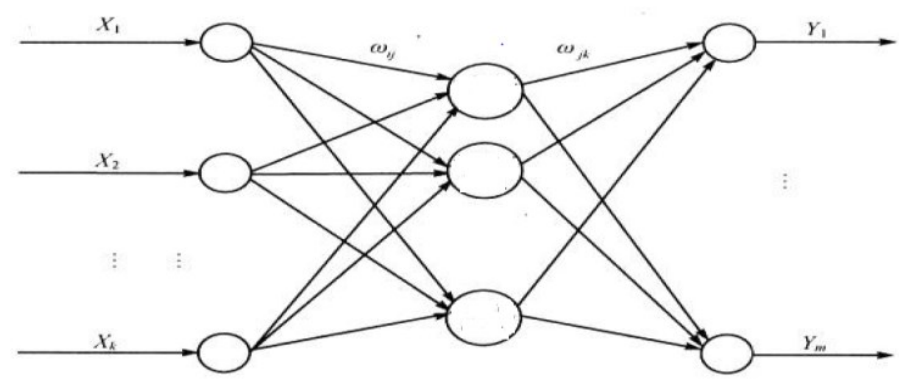

Figure 1. Wavelet neural network topology

In figure $1, X_{1}, X_{2}, \ldots, X_{k}$ are the input parameters in the wavelet neural network topology, and $\mathrm{Y}_{1}$, $\mathrm{Y}_{2}, \ldots, \mathrm{Y}_{\mathrm{m}}$ are the forecasting output. The parameters $\omega_{\mathrm{ij}}$ and $\omega_{j k}$ are the weights of wavelet neural network.

When the input sequence is $x_{i}(\mathrm{i}=1,2, \ldots, \mathrm{k})$, the output of the hidden layer can be expressed as:

$$
h(j)=h_{j}\left(\frac{\sum_{i=1}^{k} \omega_{i j} x_{i}-b_{j}}{a_{j}}\right) j=1,2, \ldots, l
$$

$h(j)$ is the result of the output of the first $\mathrm{j}$ node of the hidden layer. $\omega_{\mathrm{ij}}$ is the connection weights between the input layer and the hidden layer. $b_{j}$ is the translation factor of wavelet basis function 
$h_{j} . a_{j}$ is the expansion factor of wavelet basis function. $h_{j}$ is the wavelet basis function. In this article, the wavelet basis function we used is the Morlet function, and Morlet can be expressed as following:

$$
y=\cos (1.75 x) e^{-x^{2} / 2}
$$

Wavelet neural network output layer can be expressed as:

$$
y(k)=\sum_{i=1}^{l} \omega_{i k} h(i) \quad k=1,2, \ldots, m
$$

In the expression, $\omega_{i k}$ is the weight of the hidden layer and the output layer; $h(i)$ is the output of the first $\mathrm{i}$ hidden layer node; $l$ is the number of nodes in the hidden layer; $m$ is the number of nodes in the output layer [8].

Similar with BP neural network algorithm, the weights and parameters of the wavelet function gradient wavelet neural network is also used in amending method of the network, in order to make the result of wavelet neural network prediction close to the expected value. The wavelet neural network correction procedure is as follows:

calculation of the error of wavelet neural network:

$$
e=\sum_{k=1}^{m} y n(k)-y(k)
$$

In the expression, $y n(k)$ is the output of expectations, $y(k)$ is predicted output.

b. According to the prediction error $e$, the weights of the wavelet neural network and the basis function coefficients of the wavelet neural network are modified:

$$
\begin{gathered}
\omega_{n, k}^{(i+1)}=\omega_{n, k}^{i}+\Delta \omega_{n, k}^{(i+1)} \\
a_{k}^{(i+1)}=a_{k}^{i}+\Delta a_{k}^{(i+1)} \\
b_{k}^{(i+1)}=a_{k}^{i}+\Delta b_{k}^{(i+1)}
\end{gathered}
$$

$\Delta \omega_{n, k}^{(i+1)}, \Delta a_{k}^{(i+1)}, \Delta b_{k}^{(i+1)}$ is obtained by the prediction error of the wavelet neural network:

$$
\begin{gathered}
\Delta \omega_{n, k}^{(i+1)}=-\eta \frac{\partial e}{\partial \omega_{n, k}^{(i)}} \\
\Delta a_{k}^{(i+1)}=-\eta \frac{\partial e}{\partial a_{k}^{(i)}} \\
\Delta b_{k}^{(i+1)}=-\eta \frac{\partial e}{\partial b_{k}^{(i)}}
\end{gathered}
$$

$\eta$ is learning rate.

The steps wavelet neural network algorithm training are as following:

Step 1. Neural network initialization. Make random initialization of the expansion factor, the translation factor and the network connection weights of wavelet neural network, and set the network learning rate.

Step 2. Classification of samples. The samples are divided into training samples and test samples. The training samples are used to train the network. The test samples are used to evaluate the accuracy of the network prediction.

Step 3. To predict the output. The training samples are input to the wavelet neural network, and then calculate the output values of the neural network. Finally, the error of the output and the expected output of the neural network are calculated. 
Step 4. To modify the weight. According to the error, the weights of wavelet neural network and the parameters of wavelet function are modified, and the predicted value of the wavelet neural network is expected to the expected value.

Step 5. Judge whether the algorithm is over, if not the end, then return to step 3.

\section{Data}

The data used in this paper comes from the pollution source data from 12 enterprises in Taizhou city from December 30, 2015 to January 1, 2015. Data contains the 12 companies sulfur dioxide, nitrogen oxide concentration of the value of the data. In this paper, we will use the wavelet neural network to train and test the data of the 12 enterprises. So as to realize the analysis and prediction of pollutant concentration.

\section{Result}

Taking one of the chemical plant in January 1, 2015 to December 30, 2015 sulfur dioxide emissions as the research object, the data is normalized to [-1, 1]. From January 1, 2015 to September 30, 2015, take the concentration of sulfur dioxide as the training data. October 1, 2015 to December 31, 2015 , take the concentration of sulfur dioxide as the forecast data. Wavelet neural network will be trained 100 times, the training process of neural network prediction error change trend shown in figure 2:

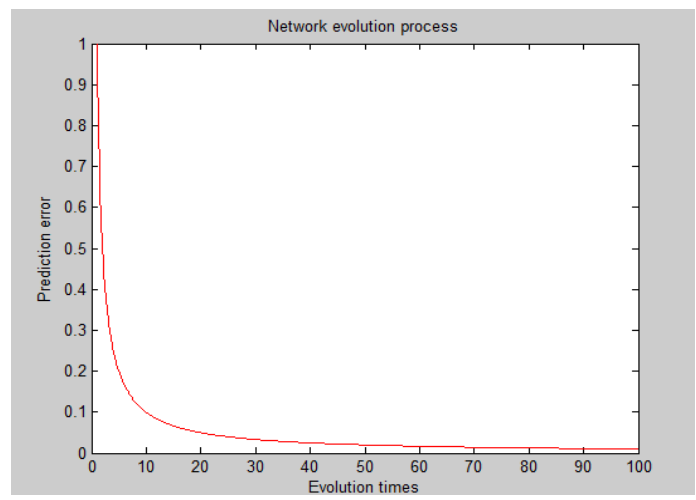

Figure 2. Network evolution process

Using the trained neural network to predict the concentration of sulfur dioxide, the predicted results and the actual value of sulfur dioxide concentration as shown in figure 3:

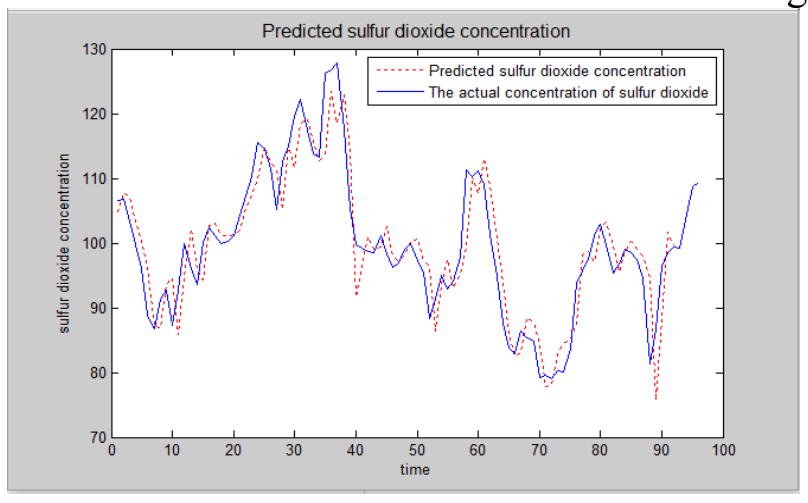

Figure 3. Comparison of sulfur dioxide concentration and the actual value of sulfur dioxide concentration

It can be seen from the results that the wavelet neural network can accurately predict the concentration of sulfur dioxide, and the network predictive value is close to the expected value. 


\section{Acknowledgments}

Project supported by The project is supported by the National Natural Science Foundation of China (Grant Nos. 61271082,31671006), Jiangsu Provincial Key R \& D Program (Social Development) (Grant No.BE2015700,BE2016773), the Natural Science Foundation of Jiangsu Province (Grant No. BK20141432),Natural Science Research Major Program in Universities of Jiangsu Province (Grant No.16KJA310002).Environmental Protection Research Project of Jiangsu Province(Grant No.2015045).

\section{References}

[1] A.L. Barabasi, E. Ravasz and T. Vicsek, "Deterministic Scale-Free Networks," Physica A, vol. 299, pp. 559-564, 15 October 2010.

[2] C. Song, S. Havlin and H. A. Makse, "Self-similarity of complex networks," Nature, pp. 392-433, 27 January 2005.

[3] J. Zhang, M. Small, "Complex network from pseudoperiodic time series: topology versus dynamics,” Phys. Rev. Lett, vol. 96, pp. 238701 -238701, 16 June 2006.

[4] T. Karagiannis, M. Molle and M. Faloutsos, "Long-Range Dependence: Ten Years of Internet Traffic Modeling,” IEEE Internet. Comput, vol. 8, pp. 57-64, October 2004.

[5] C. Song, S. Havlin and H.A. Makse, "Origins of fractality in the growth of complex networks," Nat. Phys, vol. 2, pp. 275-281, April 2006.

[6] J.H. Ma, Y.S. Chen, "An analytic and application to state space reconstruction about chaotic time series,” Appl. Math. Mech. -Engl, vol. 21, pp. 1117-1124, November 2000.

[7] K.I. Goh, G. Salvi, and B. Kahng, "Skeleton and fractal scaling in complex networks," Phys. Rev. Lett, vol. 96, October 2006.

[8] J.S. Kim, K.I. Goh and G. Salvi, "Fractality in complex networks: critical and supercritical skeletons," Phys. Rev. E, vol. 75, pp. 44-56, January 2007. 